\title{
A 20-Year-0ld Contraceptive Device Transformed into a Calcified Mass
}

\author{
Awatuf Elshirif, Seema Sadiq \\ Princess Royal University Hospital, King's College Hospital NHS Foundation Trust, London, UK \\ Email: awatuf.elshirif@nhs.net
}

Received 29 April 2014; revised 26 May 2014; accepted 3 June 2014

Copyright (C) 2014 by authors and Scientific Research Publishing Inc.

This work is licensed under the Creative Commons Attribution International License (CC BY). http://creativecommons.org/licenses/by/4.0/

c) (i) Open Access

\begin{abstract}
Case: A 52-year-old female who was presented to accident and emergency felt unwell; she reported that she had an IUCD inserted over 20 years ago. On examination she had a large calcified mass occupying the vagina. CT scan confirmed a calcified IUCD. Conclusion: Serious complication secondary to IUCD is rare but well recorded, extensive calcification as in this case is very rare and has not been previously reported.
\end{abstract}

\section{Keywords}

Calcified, IUCD, Contraceptive, Device, Mass

\section{Introduction}

The copper intrauterine contraceptive device has been largely superseded by the IUS in recent years. However, it is still a widely used contraceptive option and is the most effective non-hormonal form of birth control. The suggested duration of effectiveness ranges from three to ten years depending on the make of IUCD, however most of them are removed after around 5 years. Risks of neglecting an IUCD include migration, infection and calcification. A case of IUCD calcification, with an unusual presentation as a possible vesico-vaginal fistula is described and discussed [1]-[3].

\section{Case Report}

A 52-year-old female presented to A\&E feeling generally unwell with a decreased appetite. Her medical background included idiopathic myelopathy, which had left her bed bound due to increased tone in her lower limbs. She also suffered with epilepsy, osteoporosis, mild hypercalcemia and urinary incontinence. On examination by the A\&E team, she was found to have a necrotic ulcer on her sacral area measuring approximately $3 \mathrm{~cm}$ by $2 \mathrm{~cm}$ with surrounding erythema. She reported that the area had been red and tender for the past few weeks, for which 
she had been applying emollient but it was not improving. Her blood tests showed a CRP of 266, WCC 9.0, neutrophils 5.9, Hb 100, U\&Es and LFTs normal. Interesting her adjusted calcium was normal at 2.43. A\&E referred her to the surgical team for review with regards to debridement. This was carried out and a vac pump dressing applied. In order to maintain good hygiene a catheter was inserted. After a difficult attempt, the surgical team asked gynaecology team for a review as it was believed a vaginal prolapse might be the cause of the difficulty inserting the catheter.

She was reviewed by the gynaecology senior registrar. He documented the review of a post menopausal female, nulligravida. The patient reported that she had an IUCD inserted over 20 years ago, and this had not been removed. On examination, a large, grey mass was visualised in the anterior vaginal wall measuring approximately $8 \mathrm{~cm} \times 5 \mathrm{~cm}$ in size. The mass was fixed and hard in consistency. The cervix was not visualised due to the obstructing mass. A CT scan was requested to investigate further.

The CT scan was reported as:

There is a fairly large calcified mass occupying the vagina, with well-defined outline and measuring at least 8 $\mathrm{cm}$ in length (Figure 1, Figure 2). At the superior end of this calcified mass are a few small bubbles of gas and a small atrophic uterus with a coil in situ. On the urographic part of the study there is no opacified urine within the bladder and no obvious defect in the posterior bladder wall. There are bilateral moderate pleural effusions and a small amount of ascites around the liver. There is mild bilateral hydronephrosis and hydroureter, but no significant delay in excretion. Conclusion: large vaginal stone. IUCD at superior margin, within cavity of atrophic uterus, though the tip of the coil is embedded in the upper margin of the stone.

Following the CT scan, the urology team was asked to review to investigate the possibility of a vesico-vaginal fistula with calculus passing through the fistula. A cystogram was performed which confirmed that there was no fistula demonstrated between the bladder and the vagina, therefore this mass was originating in the vaginal area.

After review by the gynaecology consultant, a working diagnosis of a calcified mass originating from the IUCD was made. She was taken to theatre for EUA.

Examination under anesthesia revealed a friable stone in the vagina measuring at least $8 \mathrm{~cm}$ in length surrounding the IUCD, extending into the uterine cavity, the stone was removed, the vaginal and the paraurethral skin was very friable.

Hysteroscopy was performed, uterine cavity appear normal, no sign of perforation.

Close examination of the stone demonstrated calcification around a central core of the IUCD and its associated threads (Figure 3).

Calculus analysis has shown brown, hard, crystalline appearance and weighed $6958 \mathrm{mg}$, consists of predominantly magnesium ammonium phosphate (88\%) and ammonium urate (12\%).

The vaginal culture grew normal flora only with no evidence of actinomycosis.

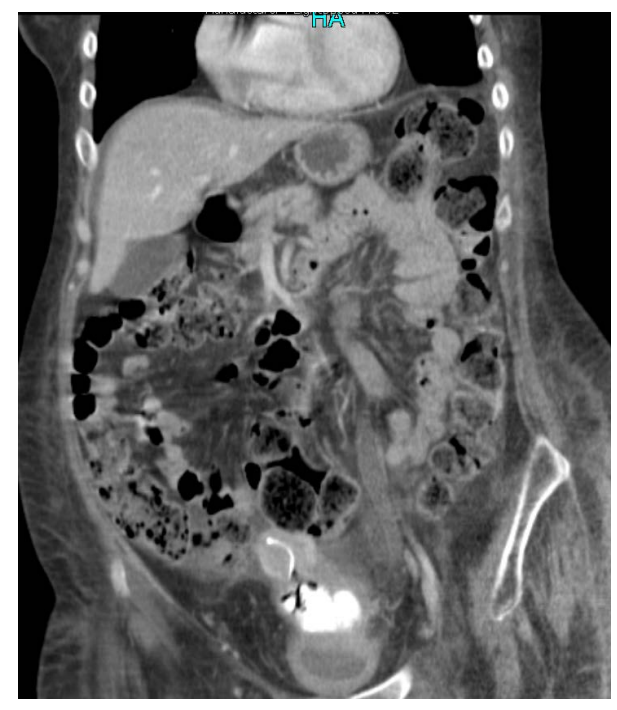

(a)

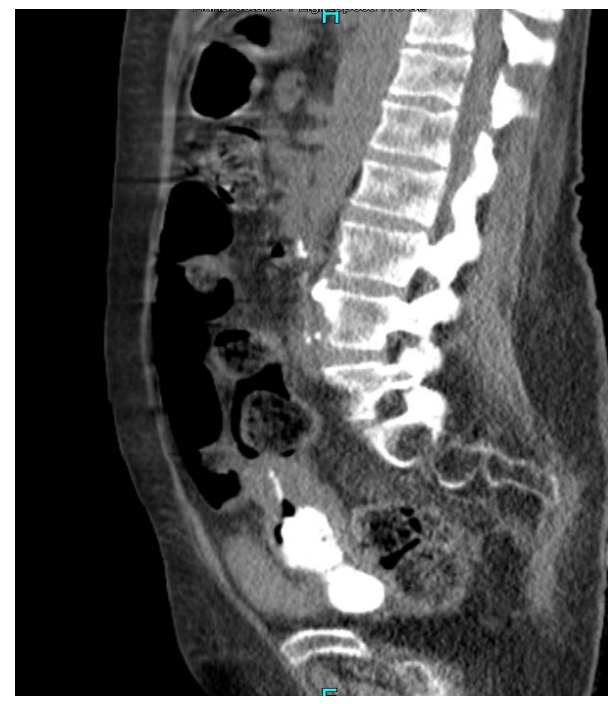

(b)

Figure 1. CT scan showing calcified mass (calcified IUCD). 


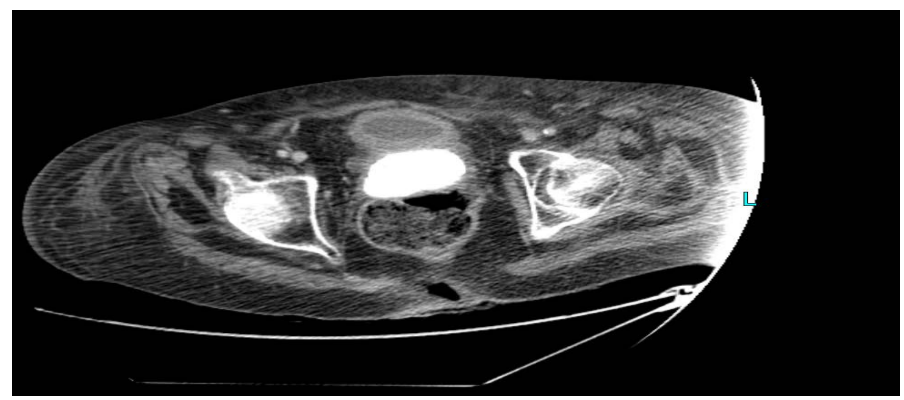

Figure 2. CT scan showing calcified mass (calcified IUCD).

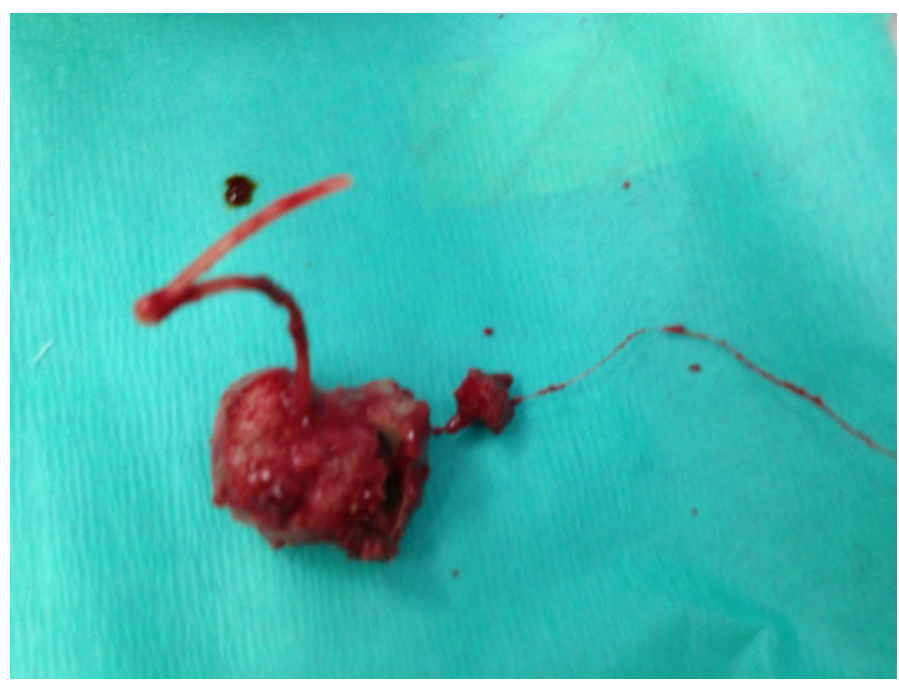

Figure 3. Calcified IUCD after removal.

\section{Discussion}

Serious complications secondary to IUCD are rare but well recorded. Perforation of the uterine fundus is the most common problem occurring in 1 in 350 to 1 in 2500 insertions [1]. Once perforation has occurred, damage has been described to most pelvic organs. Surface deposition on IUCD occurs in a linear manner with time [2] with changes detectable on electron microscopy within three months of insertion; deposition on copper containing devices occurs significantly sooner than that on inert devices [3]. Extensive encrustation as in this case has not been previously reported and is particularly unusual with the IUCD subsequently acting as a focus for calcification although no convincing evidence of vesico-vaginal fistula was ever demonstrated.

\section{References}

[1] Key, T.C. and Kreutner, A.K. (1980) Gastro-Intestinal Complications of Modern Intrauterine Devices. Obstetrics Gynecology, 55, 239-244.

[2] Rizk, M., Shaban, N., Medhat, I., et al. (1990) Electron Microscopic and Chemical Study of the Deposits Formed on Copper and Inert IUCD. Contraception, 42, 643-653. http://dx.doi.org/10.1016/0010-7824(90)90005-G

[3] Yuan, W., Shen, H., Lu, J., et al. (1986) Analysis of Surface Deposits on Intrauterine Contraceptive Devices. Advances in Contraception, 2, 193-204. http://dx.doi.org/10.1007/BF01849230 